\title{
Synthesis of chiral amino acid - derived nitrones and 1,3-dipolar cycloadditions with acrylic acid methyl ester
}

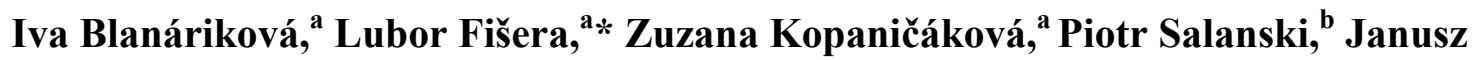 \\ Jurczak, ${ }^{b}$ and Christian Hametner ${ }^{c}$ \\ ${ }^{a}$ Department of Organic Chemistry, Slovak University of Technology, SK-812 37 Bratislava, \\ Slovak Republic \\ ${ }^{b}$ Institute of Organic Chemistry, Polish Academy of Sciences, PL-01 224 Warsaw, Poland \\ ${ }^{c}$ Institute of Organic Chemistry, University of Technology, A-1060 Vienna, Austria \\ E-mail: fisera@cvt.stuba.sk or blanarik@chelin.chtf.stuba.sk
}

To Miha Tišler on the occasion of his $75^{\text {th }}$ birthday

(received 30 Jun 05 ; accepted 23 Oct 01 ; published on the web 31 Oct 01 )

\begin{abstract}
The chiral nitrones derived from $\alpha$-amino acids react with acrylic acid methyl ester to afford the corresponding diastereomeric isoxazolidines 9a-d. The major products are in the $\mathrm{C}-3 / \mathrm{C}-4$ erythro- and $\mathrm{C}-3 / \mathrm{C}-3 \mathrm{a}$ trans-configuration. The nitrones 3-8 were prepared from the corresponding $\alpha$-amino aldehydes, starting from L-phenylalanine and L-valine.
\end{abstract}

Keywords: Cycloaddition, nitrones, isoxazolidines, $\alpha$-amino aldehydes

\section{Introduction}

The nitrone-olefin 1,3-dipolar cycloaddition is a powerful reaction in that it can create as many as three new contiguous stereogenic centers in a single step. ${ }^{1}$ Based on an evaluation of the nitrone cycloaddition, it was felt that the stereochemistry of these new centers could be controlled if the reaction system was properly designed. ${ }^{1-3}$ With our continuing efforts to utilize chiral 1,3-dipolar cycloadditions, ${ }^{4-7}$ and with the goal of developing a simple route to the synthesis of proteinogenic and non-proteinogenic amino $\operatorname{acids}^{8}$ via an asymmetric 1,3-dipolar cycloaddition we have chosen some $\alpha$-amino acid - derived nitrones 3-8 as template for a nitron cycloaddition. Diastereoselective synthesis of $\beta, \gamma$,-diamino acids has received considerable attention, this is due to wide range of biological activities of these compounds e. g. emericedines A (1a), B (1b) and C (1c) - inhibit long chain fatty acid oxidation, aminostatine (2) - substrate analogue of statine led to potent aspartyl proteinase inhibitor of humane renine. ${ }^{9}$ 
<smiles>[R]N[C@H](CC(=O)[O-])C[N+](C)(C)C</smiles>

1 a : $\mathrm{R}=\mathrm{CH}_{3} \mathrm{CO}$

b : $\mathrm{R}=\mathrm{CH}_{3} \mathrm{CH}_{2} \mathrm{CO}$

c : $\mathrm{R}=\mathrm{CH}_{3} \mathrm{CH}_{2} \mathrm{CH}_{2} \mathrm{CO}$<smiles>CCOC(=O)C[C@@H](N)[C@H](N)CC(C)C</smiles>

2

In this paper we report the synthesis of chiral nitrones derived from $\alpha$-amino acids and the stereoselectivity of the cycloaddition with acrylic acid methyl ester.

\section{Results and Discussion}

The nitrones 3-8, with a "chiral " $C$ - substituent, were prepared from the corresponding $\alpha$-amino aldehydes, ${ }^{10}$ by treatment with the $N$-benzyl hydroxylamine in dichloromethane in the presence of anhydrous magnesium sulfate, ${ }^{11}$ starting from L-phenylalanine and L-valine. A single isomer was isolated in all cases in pure state, and the expected $Z$ configuration was confirmed by nuclear Overhauser effect (NOE) difference spectroscopy. The nitrones 5, 8 were already described by $\mathrm{P}$. Merino, ${ }^{12}$ the nitrones 3, 4, 6 and 7 are originals from our laboratory. $\alpha$-Amino aldehydes are mainly obtained from $\alpha$-amino acids. ${ }^{10}$ The first synthetic approach is based on the reduction of corresponding esters or amides of $\alpha$-amino acids to aldehydes. In this work we have used the second method, the oxidation of $\alpha$-amino alcohols obtained from $\alpha$-amino acids (Scheme 1). ${ }^{13,14}$<smiles>[O-]/[N+](=C\C(Cc1ccccc1)Cc1ccccc1)Cc1ccccc1</smiles>

3<smiles>CC(C)[C@H](C=[N+]([O-])Cc1ccccc1)c1ccccc1</smiles>

6<smiles>O=C(N[C@H](/C=[N+](\[O-])Cc1ccccc1)Cc1ccccc1)OCc1ccccc1</smiles>

4<smiles>CC(C)[C@H](C=[N+]([O-])Cc1ccccc1)NC(=O)O</smiles>

7<smiles>O=C(N[C@H](C=[N+]([O-])c1ccccc1)Cc1ccccc1)OCc1ccccc1</smiles>

5<smiles>CC(C)[C@H](C=[N+]([O-])Cc1ccccc1)C(N)=O</smiles>

8 
The $N$-Boc, Cbz-protected $\alpha$-amino alcohols were prepared by $\mathrm{LiBH} 4$ or $\mathrm{NaBH} 4-\mathrm{LiCl}$ reduction of the corresponding methyl esters. TEMPO - 2,2,6,6-tetramethylpiperidin-1-yloxy radical with $\mathrm{NaOCl}$ was used as the oxidizing reagent in the next step. ${ }^{13}$ The $N$-Bn2-protected $\alpha$ amino alcohols were prepared from corresponding parent amino alcohols by the methode of Beaulieu and Wernic. ${ }^{14}$ Pyridine-sulfur trioxide was used as the oxidizing reagent in the Swern oxidation (results are given in Table 1).

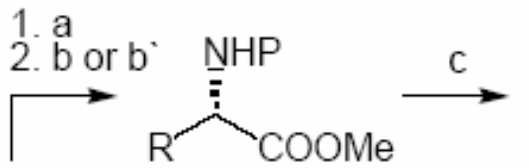<smiles>[R]C(N)C(=O)O</smiles>

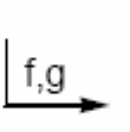<smiles>[R]C(CO)[Pb]CC</smiles><smiles>[R]C(N)CO</smiles><smiles>[R]C(N)C=O</smiles><smiles>[R]C(N)C=[N+]([O-])Br</smiles>

$4,5-7,8$

\section{Scheme 1}

Reagents and reaction conditions: (a) $\mathrm{SOCl}_{2}, \mathrm{MeOH},-78^{\circ} \mathrm{C}$; (b) $\mathrm{CbzCl}, \mathrm{NaHCO}_{3}, \mathrm{AcOEt}, 0^{\circ} \mathrm{C}$; (b') $\mathrm{Boc}_{2} \mathrm{O}, \mathrm{NaHCO}_{3}$, RT; (c) $\mathrm{LiBH}_{4}$, EtOH-THF, $0^{\circ} \mathrm{C}$; (d) TEMPO, $\mathrm{NaOCl}, \mathrm{NaBr}, \mathrm{NaHCO}_{3}$, $\mathrm{H}_{2} \mathrm{O}$, toluene AcOEt, $0^{\circ} \mathrm{C}$; (e) $\mathrm{BnNHOH}, \mathrm{MgSO}_{4}, \mathrm{CH}_{2} \mathrm{Cl}_{2}$, RT; (f) $\mathrm{NaBH}_{4}, \mathrm{H}_{2} \mathrm{SO}_{4}, \mathrm{RT}$; (g) $\mathrm{BnBr}$, $\mathrm{K}_{2} \mathrm{CO}_{3}, \mathrm{EtOH} ;$ (h) Pyr. $\mathrm{SO}_{3}, \mathrm{DMSO}, \mathrm{Et}_{3} \mathrm{~N}, 15^{\circ} \mathrm{C}$.

Table 1. Synthesis of chiral amino acid - derived nitrones 3-8

\begin{tabular}{cccccc}
\hline Entry & Nitrone & $\alpha$-Amino Acid & $\mathrm{NH}_{2}$ - Protecting group & Yield \% & Yield $\%^{2}$ \\
\hline 1 & $\mathbf{3}$ & L-phenylalanine & $\mathrm{Bn}_{2}$ & 86 & 87 \\
2 & $\mathbf{4}$ & L-phenylalanine & $\mathrm{Cbz}$ & 67 & 85 \\
3 & $\mathbf{5}$ & L-phenylalanine & $\mathrm{Boc}$ & 70 & 83 \\
4 & $\mathbf{6}$ & L-valine & $\mathrm{Bn}_{2}$ & 72 & 80 \\
5 & 7 & L-valine & $\mathrm{Cbz}$ & 65 & 79 \\
6 & $\mathbf{8}$ & L-valine & $\mathrm{Boc}$ & 71 & 77 \\
\hline
\end{tabular}

${ }^{1}$ Overall yield from amino acid to amino alcohol. ${ }^{2}$ Overall yield from amino alcohol to nitrone. 
Next, we report some examples of the 1,3-dipolar cycloaddition of chiral nitrones 3-8 with acrylic acid methyl ester with purpose to apply their cycloadducts to the synthesis of $\gamma$ substituted $\beta, \gamma$-diamino acids (Scheme 2). Our objective was the study of diastereoselectivity of cycloadditions stemming from the nitrone part. The cycloadditions afforded the corresponding diastereomeric isoxazolidines 9a-d as a mixture of four diastereoisomers in a good overall yield (Table 2). The regioselectivity of cycloaddition was high, indeed 5-substituted isoxazolidines were formed exclusively. The stereoselectivity of cycloaddition was dependent on minor structural changes in starting nitrone as well as to the reaction conditions (refluxing toluene or at high pressure, ${ }^{15} 10 \mathrm{Kbar}$ in toluene at $60^{\circ} \mathrm{C}$ or RT, Table 2).

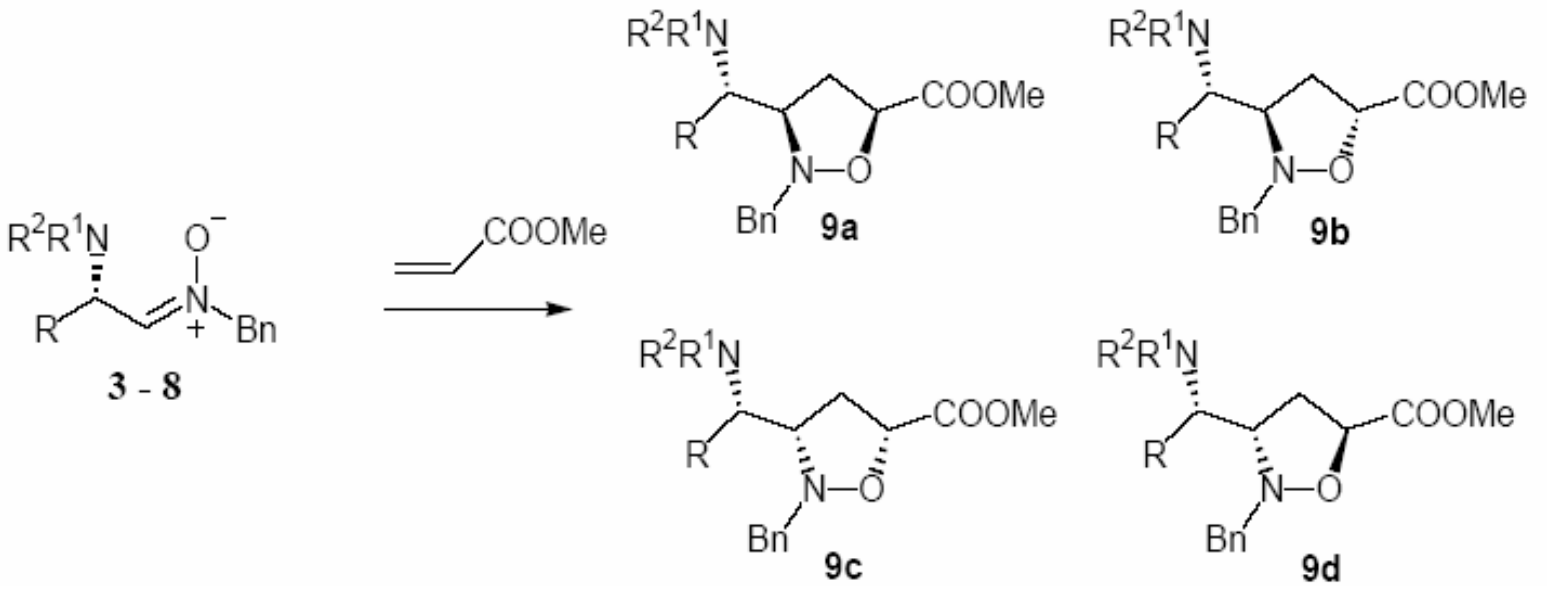

\section{Scheme 2}

Table 2. 1,3-Dipolar cycloaddition of chiral amino acid - derived nitrones to acrylic acid methyl ester

\begin{tabular}{|c|c|c|c|c|}
\hline Entry & Nitrone & Reaction condition & Ratio $^{a}$ & Yield \% \\
\hline 1 & 3 & RT, 1.5 d., toluene, $10 \mathrm{Kbar}$ & $62: 14: 13: 11$ & 94 \\
\hline 2 & 3 & $60^{\circ} \mathrm{C}, 1.5 \mathrm{~d}$., toluene, $10 \mathrm{Kbar}$ & $60: 16: 14: 10$ & 90 \\
\hline 3 & 3 & $110^{\circ} \mathrm{C}, 1 \mathrm{~h} .$, toluene & $60: 15: 13: 12$ & 89 \\
\hline 4 & 4 & RT, 2 d., $\mathrm{CH}_{2} \mathrm{Cl}_{2}, 10 \mathrm{Kbar}$ & $45: 26: 19: 10$ & 87 \\
\hline 5 & 4 & $60{ }^{\circ} \mathrm{C}, 2 \mathrm{~d} ., \mathrm{CH}_{2} \mathrm{Cl}_{2}, 10 \mathrm{Kbar}$ & $48: 24: 21: 6$ & 82 \\
\hline 6 & 4 & $60^{\circ} \mathrm{C}, 2 \mathrm{~d}$., toluene, $10 \mathrm{Kbar}$ & $51: 21: 21: 7$ & 89 \\
\hline 7 & 5 & RT, 2 d., toluene, 10 Kbar & $47: 27: 13: 13$ & 76 \\
\hline 8 & 5 & $60^{\circ} \mathrm{C}, 2 \mathrm{~d}$., toluene, $10 \mathrm{Kbar}$ & $50: 28: 12: 10$ & 78 \\
\hline 9 & 5 & $60^{\circ} \mathrm{C}, 2$ d., $\mathrm{CH}_{2} \mathrm{Cl}_{2}, 10 \mathrm{Kbar}$ & 47:-:29:24 & 80 \\
\hline 10 & 5 & $110^{\circ} \mathrm{C}, 1 \mathrm{~h} .$, toluene & $58: 21: 11: 10$ & 88 \\
\hline 11 & 6 & RT, 2 d., toluene, $10 \mathrm{Kbar}$ & $54: 19: 14: 13$ & 84 \\
\hline
\end{tabular}


Table 2. Continued

\begin{tabular}{lllll}
\hline 12 & $\mathbf{6}$ & $60{ }^{\circ} \mathrm{C}, 2 \mathrm{~d} .$, toluene, $10 \mathrm{Kbar}$ & $52: 20: 15: 13$ & 80 \\
13 & $\mathbf{6}$ & $110^{\circ} \mathrm{C}, 1 \mathrm{~h} .$, toluene & $49: 25: 15: 11$ & 90 \\
14 & 7 & $\mathrm{RT}, 2 \mathrm{~d} ., \mathrm{CH}_{2} \mathrm{C}_{2}, 10 \mathrm{Kbar}$ & $57: 31: 7: 5$ & 85 \\
15 & $\mathbf{8}$ & $110^{\circ} \mathrm{C}, 1 \mathrm{~h} .$, toluene & $53: 27: 12: 8$ & 92 \\
\hline
\end{tabular}

${ }^{\mathrm{a}}$ The ratio of diastereoisomers was determined from ${ }^{13} \mathrm{C}$ NMR spectra

The detailed study of the stereoselectivity of these cycloadditions and elucidation of the structures of the obtained cycloadducts is in progress.

\section{Experimental Section}

General Procedures. All starting materials and reagents are commercially available (Fluka, Merck or Avocado) and were used without further purification. Solvents were dried before use. Thin-layer chromatography (TLC, on aluminium plates coated with silica $60 \mathrm{~F}_{254}, 0.25 \mathrm{~mm}$ thickness, Merck) was used for monitoring of reaction courses; eluents are given in the text. For column chromatography done the flash chromatography technique was employed using silica 60 (0.040 - $0.063 \mathrm{~mm}$, Merck). Melting points (m.p.) were determined on a Kofler hot plate apparatus. The ${ }^{1} \mathrm{H}$ and ${ }^{13} \mathrm{C}$ NMR spectra of deuterochloroform solutions were obtained using Varian VXR $300(300 \mathrm{MHz})$ and Bruker DRX-400 (400 MHz) instruments, tetramethylsilane being the internal reference. Optical rotations $[\alpha]$ were measured on an IBZ Messtechnik Polar$\mathrm{L} \mu \mathrm{P}$ polarimeter at the sodium D line $(589 \mathrm{~nm})$ using a $1 \mathrm{dm}$ cell with chloroform as solvent. $N$ Protected alcohols were prepared according to the already published procedures. analytical and spectral data for $N$-protected alcohols were identical with those already published. ${ }^{14}$

\section{TEMPO oxidation of alcohols to aldehydes. General procedure}

To a cold $\left(0^{\circ} \mathrm{C}\right)$, rapidly stirred biphasic mixture consisted of an alcohol (1 mmol), TEMPO free radical $(0.02 \mathrm{mmol}), \mathrm{NaBr}(1 \mathrm{mmol})$, toluene $(3 \mathrm{~mL})$, ethyl acetate $(3 \mathrm{~mL})$ and water $(0.5 \mathrm{~mL})$, an aqueous solution of $\mathrm{NaOCl}(1.1 \mathrm{mmol})$ containing $\mathrm{NaHCO}_{3}(2.9 \mathrm{mmol})$ was added dropwise over a period of 1-2 h. The aqueous layer was separated and washed with $\mathrm{Et}_{2} \mathrm{O}(5 \mathrm{~mL})$. The combined organic layers were washed with a solution of $\mathrm{KI}(8 \mathrm{mg})$, dissolved in $10 \%$ aqueous $\mathrm{KHSO}_{4}(2 \mathrm{~mL})$, then with $10 \%$ aqueous sodium thiosulfate $(1 \mathrm{~mL})$, brine $(2 \mathrm{~mL})$ and dried $\left(\mathrm{Na}_{2} \mathrm{SO}_{4}\right)$. Filtration and concentration in vacuo afforded a desired aldehyde which was immediately used for further reactions. 


\section{Swern oxidation of alcohols to aldehydes. General procedure}

By the method of Beaulieu and Wernic, pyridine-sulfur trioxide complex $(1.7 \mathrm{mmol})$ in DMSO $(10 \mathrm{~mL})$ was added in small portions over 10-20 mins to a solution of an alcohol $(1 \mathrm{mmol})$ and $\mathrm{Et}_{3} \mathrm{~N}(1.9 \mathrm{mmol})$ in DMSO $(10 \mathrm{~mL})$ at $0{ }^{\circ} \mathrm{C}$. The mixture was allowed to warm to $15{ }^{\circ} \mathrm{C}$, and after $2 \mathrm{~h}$ the reaction was quenched with ice water $(20 \mathrm{~mL})$ and extract with $\mathrm{CH}_{2} \mathrm{Cl}_{2}(3 \mathrm{x} 15 \mathrm{~mL})$. The extracts were washed with water $(20 \mathrm{~mL})$, dried $\left(\mathrm{Na}_{2} \mathrm{SO}_{4}\right)$, filtered and concentrated under reduced pressure to give a crude aldehyde, which was immediately used for further reactions.

\section{Synthesis of $\alpha$-amino nitrones. General procedure}

To a well-stirred solution of the corresponding $\alpha$-amino aldehyde $(1 \mathrm{mmol})$ in dichloromethane $(10 \mathrm{~mL})$, anhydrous magnesium sulfate $(1.5 \mathrm{mmol})$ and $N$-benzylhydroxylamine $(1 \mathrm{mmol})$ were added sequentially and resulting mixture wasstirred at RT overnight. Filtration and concentration in vacuo afforded a crude product which was purified by columm chromathography on silica gel to yield the pure product (eluent : EtOAc/i-hexane).

(Z)- $N$-[(2S)-2-(Benzyloxycarbonylamino)-3-phenylpropylidene] benzylamine $\boldsymbol{N}$-oxide (4). Colourless crystals m. p. $=145-147{ }^{\circ} \mathrm{C},[\alpha]_{\mathrm{D}}{ }^{25}=+25.5\left(\mathrm{c}=1.0, \mathrm{CHCl}_{3}\right), \mathrm{R} f=0.18(\mathrm{EtOAc} / \mathrm{i}-$ hexane 50:50). ${ }^{1} \mathrm{H}$ NMR $\left(\mathrm{CDCl}_{3}, 200 \mathrm{MHz}\right): \delta 3.15$ (m, 2H, H-3), $4.75(\mathrm{~m}, 1 \mathrm{H}, \mathrm{H}-2), 4.85(\mathrm{~s}, 2 \mathrm{H}$, $\mathrm{COOCH}_{2} \mathrm{Ph}, 5.10$ (s, 2H, NCH$\left.{ }_{2} \mathrm{Ph}\right), 6.28$ (d, J=6.4 Hz, 1H, H-NH), 6.76 (d, J=5.3 Hz, 1H, H-

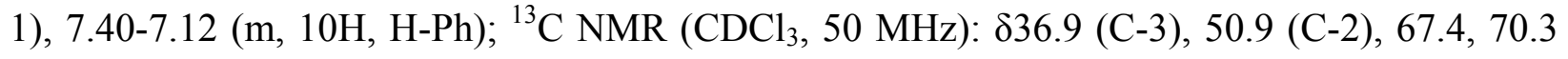
(2C- $\left.\mathrm{CH}_{2} \mathrm{Ph}\right), 127.3,128.5,128.6,129.0,129.1,129.5,129.7,129.9$ (15C, 2-6CPh), $132.8(\mathrm{C}-1)$, 136.9 (C, 1-CPh), 137.5 (2C, 1-CPh), $156.3(\mathrm{C}=\mathrm{O})$.

(Z)- $\mathrm{N}$-[(2S)-2-(tert-Butoxycarbonylamino)-3-phenylpropylidene] benzylamine $\boldsymbol{N}$-oxide (5). Colourless crystals m. p. $=128-130{ }^{\circ} \mathrm{C},[\alpha]_{\mathrm{D}}{ }^{25}=+32.6\left(\mathrm{c}=1.0, \mathrm{CHCl}_{3}\right), \mathrm{R} f=0.22(\mathrm{EtOAc} / \mathrm{i}-$ hexane 50:50). ${ }^{1} \mathrm{H} \mathrm{NMR}\left(\mathrm{CDCl}_{3}, 200 \mathrm{MHz}\right): \delta 1.43\left(\mathrm{~s}, 9 \mathrm{H}, \mathrm{C}\left(\mathrm{CH}_{3}\right)_{3}\right), 3.14(\mathrm{~m}, 2 \mathrm{H}, \mathrm{H}-3), 4.68$ (ddd, $J=7.3,6.7,5.7 \mathrm{~Hz}, 1 \mathrm{H}, \mathrm{H}-2), 4.87$ (s, 2H, $\left.\mathrm{CH}_{2} \mathrm{Ph}\right), 5.95$ (bs, $\left.1 \mathrm{H}, \mathrm{H}-\mathrm{NH}\right), 6.75$ (d, $J=5.7$ $\mathrm{Hz}, 1 \mathrm{H}, \mathrm{H}-1), 7.40,7.27,7.14(3 \mathrm{xm}, 10 \mathrm{H}, \mathrm{H}-\mathrm{Ph}) ;{ }^{13} \mathrm{C} \mathrm{NMR}\left(\mathrm{CDCl}_{3}, 50 \mathrm{MHz}\right): \delta 20.3\left(\mathrm{C}\left(\mathrm{CH}_{3}\right)_{3}\right)$, 36.9 (C-3), $50.5(\mathrm{C}-2), 70.3\left(\mathrm{C}-\mathrm{CH}_{2} \mathrm{Ph}\right), 80.3\left(\mathrm{C}\left(\mathrm{CH}_{3}\right)_{3}\right), 127.2,129.0,129.4,129.6,129.7$, 129.9(10C, 2-6CPh), 132.9 (C-1), 137.8, 138.0 (2C, 1-CPh), $155.4(\mathrm{C}=\mathrm{O})$.

(Z)- $N$-[(2S)-2,2-(Dibenzylamino)-3-phenylpropylidene] benzylamine $\boldsymbol{N}$-oxide (3). Colourless crystals m. p. $=109-110{ }^{\circ} \mathrm{C},[\alpha]_{\mathrm{D}}{ }^{25}=+100.5\left(\mathrm{c}=1.0, \mathrm{CHCl}_{3}\right), \mathrm{R} f=0.26(\mathrm{EtOAc} / \mathrm{i}$-hexane 50:50). ${ }^{1} \mathrm{H}$ NMR $\left(\mathrm{CDCl}_{3}, 400 \mathrm{MHz}\right): \delta 2.94,3.07$ (2 x dd, $J=8.5,6.7,14.0 \mathrm{~Hz}, 2$ x 1H, H-3), $3.63,3.87\left(2 \times \mathrm{d}, J=13.7 \mathrm{~Hz}, 2 \times 2 \mathrm{H}, 2 \times \mathrm{CH}_{2} \mathrm{Ph}\right), 4.55$ (" $\mathrm{q}^{\text {“c, }}$ ddd, $\left.J=7.0 \mathrm{~Hz}, 1 \mathrm{H}, \mathrm{H}-2\right), 4.85$ (2 $\left.\mathrm{x} \mathrm{d}, J=13.4 \mathrm{~Hz}, 2 \mathrm{H}, \mathrm{CH}_{2} \mathrm{Ph}\right), 6.77$ (d, $\left.J=7.3 \mathrm{~Hz}, 1 \mathrm{H}, \mathrm{H}-1\right), 7.41-7.12(\mathrm{~m}, 20 \mathrm{H}, \mathrm{H}-\mathrm{Ph}) ;{ }^{13} \mathrm{C}$ NMR (CDCl3, $100 \mathrm{MHz}): \delta 35.9(\mathrm{C}-3), 55.5\left(2 \mathrm{C}-\mathrm{CH}_{2} \mathrm{Ph}\right) 57.5$ (C-2), $70.3\left(\mathrm{C}-\mathrm{CH}_{2} \mathrm{Ph}\right), 126.6$, $127.4,128.6,128.7,128.8,129.1,129.3,129.4,129.5,129.7,129.9\left(20 \mathrm{C}, 2-6 \mathrm{C}_{\mathrm{Ph}}\right), 133.4(\mathrm{C}-1)$, $138.7\left(2 \mathrm{C}, 1-\mathrm{C}_{\mathrm{Ph}}\right), 139.7\left(2 \mathrm{C}, 1-\mathrm{C}_{\mathrm{Ph}}\right)$.

(Z)- $\mathrm{N}$-[(2S)-2-(Benzyloxycarbonylamino)-3-methylbutylidene] benzylamine $\mathbf{N}$-oxide (7). Colourless crystals, m. p. $=153-154{ }^{\circ} \mathrm{C},[\alpha]_{\mathrm{D}}{ }^{25}=+12.8\left(\mathrm{c}=0.5, \mathrm{CHCl}_{3}\right), \mathrm{R} f=0.10(\mathrm{EtOAc} / \mathrm{i}-$ 
hexane 50:50) ${ }^{1} \mathrm{H} \mathrm{NMR}\left(\mathrm{CDCl}_{3}, 200 \mathrm{MHz}\right): \delta 0.95,0.92\left(2 \mathrm{xd}, J=7.9 \mathrm{~Hz}, 2 \times 3 \mathrm{H}, \mathrm{H}-4,4^{\prime}\right), 2.39$ (m, 1H, H-3), 4.26 (ddd, $J=7.9,5.5,8.9 \mathrm{~Hz} 1 \mathrm{H}, \mathrm{H}-2), 4.90,5.12$ (2 x s, 2 x 2H, 2 x $\mathrm{CH}_{2} \mathrm{Ph}$ ), 6.55 (d, $J=8.9 \mathrm{~Hz}, 1 \mathrm{H}, \mathrm{H}-\mathrm{NH}), 6.85$ (d, $J=5.5 \mathrm{~Hz}, 1 \mathrm{H}, \mathrm{H}-1), 7.41-7.36$ (m, $10 \mathrm{H}, \mathrm{H}-\mathrm{Ph}) ;{ }^{13} \mathrm{C}$ NMR (CDCl 3 , 50 MHz): $\delta$ 19.7, 20.2 (2C-4, 4'), 30.8 (C3), 55.3 (C-2), 67.4, $70.5\left(2 \mathrm{C}-\mathrm{CH}_{2} \mathrm{Ph}\right)$, 128.5, 128.9, 129.6, 129.7, 129.9 (10C, 2-6CPh), 132.8 (C-1), 137.4, 137.5 (2C, 1-CPh), 156.3 $(\mathrm{C}=\mathrm{O})$.

(Z)- $N$-[(2S)-2-( tert-Butoxycarbonylamino)-3-methylbutylidene] benzylamine $\boldsymbol{N}$-oxide (8). Colourless crystals, m. p. $=98-100{ }^{\circ} \mathrm{C},[\alpha]_{\mathrm{D}}{ }^{25}=+15.7\left(\mathrm{c}=1.0, \mathrm{CHCl}_{3}\right), \mathrm{R} f=0.12(\mathrm{EtOAc} / \mathrm{i}-$ hexane 50:50) ${ }^{1} \mathrm{H} \mathrm{NMR}\left(\mathrm{CDCl}_{3}, 200 \mathrm{MHz}\right): \delta 0.87,0.91\left(2 \mathrm{xd}, J=6.8 \mathrm{~Hz}, 2 \mathrm{x} \mathrm{3H}, \mathrm{H}-4,4^{\prime}\right), 1.41$ $\left(\mathrm{s}, 9 \mathrm{H}, \mathrm{H}-\mathrm{C}\left(\mathrm{CH}_{3}\right)_{3}\right), 2.30(\mathrm{~m}, 1 \mathrm{H}, \mathrm{H}-3), 4.16$ (m, 1H, H-2), 4.87 (s, 2H, $\left.\mathrm{CH}_{2} \mathrm{Ph}\right), 6.18$ (bs, 1H, $\mathrm{H}-\mathrm{NH}), 6.85$ (d, $J=5.4 \mathrm{~Hz}, 1 \mathrm{H}, \mathrm{H}-1), 7.38(\mathrm{~m}, 15 \mathrm{H}, \mathrm{H}-\mathrm{Ph}) ;{ }^{13} \mathrm{C} \mathrm{NMR}\left(\mathrm{CDCl}_{3}, 50 \mathrm{MHz}\right): \delta 19.7$,

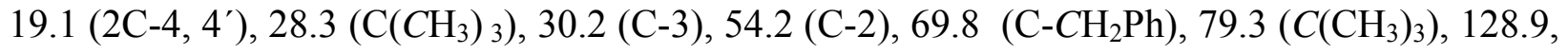
129.2, (5C, 2-6 $\left.\mathrm{C}_{\mathrm{Ph}}\right), 132.7(\mathrm{C}-1), 137.7\left(\mathrm{C}, 1 \mathrm{C}_{\mathrm{Ph}}\right), 155.8(\mathrm{C}=\mathrm{O})$.

(Z)- $\mathrm{N}$-[(2S)-2-(Dibenzylamino)-3-methylbutylidene] benzylamine $\mathrm{N}$-oxide (6). Colourless crystals, m. p. $=102-104{ }^{\circ} \mathrm{C},[\alpha]_{\mathrm{D}}{ }^{25}=-26.0,\left(\mathrm{c}=1.0, \mathrm{CHCl}_{3}\right), \mathrm{R} f=0.13($ EtOAc/i-hexane 50:50) ${ }^{1} \mathrm{H}$ NMR $\left(\mathrm{CDCl}_{3}, 200 \mathrm{MHz}\right): \delta 0.77,1.03(2 \mathrm{xd}, J=6.7 \mathrm{~Hz}, 2$ x 3H, H-4,4'), 1.90 (m, 1H, H-3), 3.49, 3.80 ( $2 \times$ d, $J=13.4 \mathrm{~Hz}, 2 \times 2 \mathrm{H}, 2 \times \mathrm{CH}_{2} \mathrm{Ph}$ ), 3.89 ("“t", dd, $J=9.6 \mathrm{~Hz}, 1 \mathrm{H}, \mathrm{H}-2$ ), 4.96 (s, $\left.2 \mathrm{H}, \mathrm{CH}_{2} \mathrm{Ph}\right), 6.73$ (d, $\left.J=8.8 \mathrm{~Hz}, 1 \mathrm{H}, \mathrm{H}-1\right), 7.40$ (m, 15H, H-Ph); ${ }^{13} \mathrm{C} \mathrm{NMR}\left(\mathrm{CDCl}_{3}, 50 \mathrm{MHz}\right): \delta$ 19.5, 20.7 (2C-4, 4'), $30.2(\mathrm{C}-3), 55.5\left(2 \mathrm{C}-\mathrm{CH}_{2} \mathrm{Ph}\right) 61.2(\mathrm{C}-2), 70.8\left(\mathrm{C}-\mathrm{CH}_{2} \mathrm{Ph}\right), 126.9,127.3$, $128.5,128.9,129.0,129.3,129.4,129.9\left(15 \mathrm{C}, 2-6 \mathrm{C}_{\mathrm{Ph}}\right), 134.6\left(\mathrm{C}, 1-\mathrm{C}_{\mathrm{Ph}}\right) 140.4(\mathrm{C}-1)$.

\section{General procedure for high pressure cycloadditions ${ }^{15}$}

Reactions of nitrones with dipolarophiles were carried out at RT or $60{ }^{\circ} \mathrm{C}$ in toluene or in $\mathrm{CH}_{2} \mathrm{Cl}_{2}$ for several days under $10 \mathrm{Kbar}$ pressure. The reaction mixture $(1 \mathrm{mmol}$ of nitrone, $2 \mathrm{mmol}$ of dipolarophile and $0.5 \mathrm{~mL}$ of solvent ) was placed in a Teflone ampoule which was inserted into the high-pressure vessel filled with hexane as a transmission medium. After decompression, the mixture was purified by flash columm chromathography.

\section{General procedure for atmospheric pressure cycloadditions}

To a stirred solution of the nitrone 1a $(1.0 \mathrm{mmol})$ in toluene $(5 \mathrm{~mL})$ was added acrylic acid methyl ester, $(2 \mathrm{mmol})$, and the solution was heated at reflux $\left(110{ }^{\circ} \mathrm{C}\right)$ for $2 \mathrm{~h}$. The resulting mixture was evaporated under reduced pressure. The crude mixture was purified and separated by column chromatography on silica gel. 


\section{Acknowledgements}

The authors are grateful to the Slovak Grant Agency (No. 1/7314/20).

\section{References}

1. (a) Tufariello, J. J. In 1,3-Dipolar Cycloaddition Chemistry, Padwa, A. Ed. Wiley Interscience: New York, 1984, Ch. 9, p 83. (b) Torssell, K.B. G. Nitrile Oxides, Nitrones, and Nitronates, VCH Publishers Inc.: New York 1988. (c) Gothelf, K.V.; Jorgensen, K.V. Chem. Rev. 1988, 98, 863.

2. Frederickson, M. Tetrahedron 1997, 53, 403.

3. Baggiolini, E. G.; Iacobelli, J. A.; Hennesy, B. M.; Batcho, A. D.; Sereno, J. F.; Uskovic, M. R. J. Org. Chem. 1986, 51, 3098. (b) Brandi, A.; Cicchi, S.; Goti, A.; Pietrusiewicz, K. M. Tetrahedron: Asymmetry 1991, 2, 1063. (c) Saito, S.; Ishikawa, T.; Kishimoto, N.; Kohara, T.; Moriwake, T. Synlett 1994, 282. (d) Kametani, T.; Chu, S. D.; Honda, T. Heterocycles 1987, 25, 241. (e) Merino, P.; Castillo, E.; Franco, S.; Merchan, F. L.; Tejero, T. J. Org. Chem. 1988, 63, 2371. (f) Bernet, B.; Vasella, A. Helv. Chim. Acta. 1979, 62, 2411.

4. Fišera, L.; Al-Timari, U. A. R.; Ertl, P. In Cycloadditions in Carbohydrate Chemistry. ACS Monograph. Am. Chem. Soc.: Washington 1992, p 158.

5. (a) Al-Timari, U. A. R.; Fišera, L.; Ertl, P.; Goljer, I.; Prónayová, N. Monatsh. Chem. 1992, 123, 999. (b) Kubán, J.; Blanáriková, I.; Fišera, L.; Prónayová, N. Chem. Papers 1997, 51, 378.

6. (a) Kubán, J.; Blanáriková, I.; Fengler-Veith, M.; Jäger, V.; Fišera, L. Chem. Papers 1998, 52, 780. (b) Blanáriková I., Dugovič B., Fišera L., Hametner C. ARKIVOC 2001, 2, 1091.

7. Kubán, J.; Blanáriková, I.; Fišera, L.; Jarošková, L.; Fengler-Veith, M.; Jäger, V.; Kozíšek, J.; Humpa, O.; Langer, V. Tetrahedron 1999, 55, 9501.

8. Jones, J. H. Amino Acids and Peptides, Specialist Periodical Report, The Royal Society of Chemistry, 1992; Vol. 2.

9. (a) Shinigawa S., Tanamura T., Harada S., Asai M., Okazaki H., J. Med. Chem., 1984, 30, 1458. (b) Arrowsmith R. J., Carter K., Dann J. G., Davies D. E., Harris C. J., Morton J. A., Lister P., Robinson J. A., Williams D. J. J. Chem. Soc., Chem. Com. 1986, 755.

10. (a) Jurczak, J., Golebiowski, A. Chem. Rev. 1989, 89, 149. (b) Reetz, M., T. Chem. Rew. 1999, 99, 1121.

11. Dondoni, A.; Franco, S.; Junquera, F.; Merchán, F.; Merino, P.; Tejero, T. Synth. Commun. 1994, 24, 2537.

12. Merino, P.; Lanaspa, A.; Merchan, F. L.; Tejero, T. Tetrahedron: Asymmetry 1997, 8, 2381.

13. Jurczak, J.; Gryko, D.; Kobrycka, A.; Gruza, H.; Prokopowicz, P. Tetrahedron 1998, 54, 
6051.

14. (a) Stanfield, C. F.; Parker, J. E.; Kanellis, P. J. Org. Chem. 1981, 46, 4799. (b) Beaulieu, P. L. Wernic, D. J. Org. Chem. 1996, 61, 3635.

15. Cardona, F.; Salanski, P.; Chmielewski, M.; Valencia, S.; Goti, A.; Brandi, A. Synlett 1998, 1444. 